$16^{\text {th }}$ International Conference on

AEROSPACE SCIENCES \& AVIATION TECHNOLOGY,

$\boldsymbol{A S A T}$ - 16 - May 26 - 28, 2015, E-Mail: asat@mtc.edu.eg

Military Technical College, Kobry Elkobbah, Cairo, Egypt

Tel : +(202) 24025292 - 24036138, Fax: +(202) 22621908

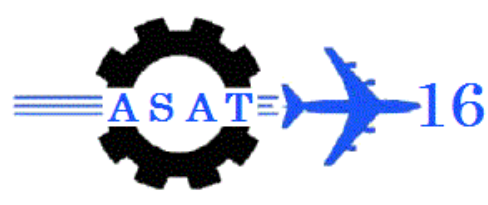

\title{
Numerical Study of Mixing Flow Regimes Generated by Different Coaxial Jet Geometries
}

M. S. Nour-Eldin", I. Elbadawy**, M. Eltelbany

Department of Mechanical Power Engineering, Faculty of Engineering at El-Mattaria, Helwan University, Masaken El-Helmia P.O., Cairo 11718, Egypt

\begin{abstract}
A computational investigation of turbulent mixing of free two air-air coaxial jets with equal densities, annular to core area ratio of 3.5 and velocity ratio of 0.5 is carried out. The flow field is considered incompressible, steady, asymmetric and turbulent. The inner and outer Reynolds numbers, based on mass averaged velocity and equivalent diameters are $8 \times 10^{4}$ and $7.5 \times 10^{4}$. Mixing flow characteristics of two different non-circular coaxial jets are obtained and compared with traditional circular coaxial jet within a relatively short length of $\mathrm{X} / \mathrm{Di}=30$. Shape and thickness of the separating wall depend on the geometry of the two coaxial jet; to maintain the AR equal to 3.5. Mixing of free jets is effective in generating the turbulence that improves the combustion and premixed flame burners performance. The simulation is performed using a finite volume scheme and a two-equation $(k-\varepsilon)$ turbulence model. Results show that velocity decay along the centerline for non-circular coaxial jet is lower than that of circular coaxial jet. Also, inner potential core of non-circular cases is greater than that of circular one. In addition, the reattachment point in case of non-circular coaxial jet is reached after that of circular coaxial jet.
\end{abstract}

Keywords: Coaxial jet, near-field region, jet decay, fully merged zone, reattachment point.

*Corresponding author. E-mail address: eng.msn.soliman@ @otmail.com

** Corresponding author. E-mail address: Ibrahim_Mohamed01@m-eng.helwan.edu.eg 


\section{INTRODUCTION}

Coaxial jets are a vital part of many engineering applications where mixing of streams of different fluids are required. They are widely used to mix the fuel and oxidizers inside the combustors of gas turbine power plant of aircraft. A properly designed jet will be desired to mix efficiently, while providing the best overall combustion efficiencies.

The flow field structure of the coaxial jet is considerably complex. Figure 1( $a$ and $b$ ) shows a schematically and simulated typical flow field of coaxial jet regions as proposed by Ko and Kwan [1], where, in their work, they divided the development or merging zone of the coaxial jets into three zones. The zone which is nearest to the nozzle exit and ends roughly at the place where the secondary or outer potential core disappears is called the initial merging zone. The termination of this zone depends on the mean velocity ratio. Immediately downstream from the initial merging zone is the intermediate zone, where the primary potential core still exists. The reattachment point ends the intermediate zone and starts the fully merged zone which is the one downstream from the intermediate zone and the last of the three zones.

Far from the nozzle, say several tens inner diameters away, the structure of the double jet is the same as that of a simple jet [2]. This region is characterized by self-similar profiles, this means that the profiles of a flow quantity, such as the axial mean velocity, taken at different downstream distances, will all collapse when properly scaled [3].

During the past few decades various experimental studies have been carried out to investigate the characteristics of coaxial jets; mainly focusing on mixing of both passive and active scalars, the shear layers between the inner and outer jets and the influence of the inlet conditions on mixing properties. For example, Forstall and Shapiro [4] studied the effects of different inlet velocity ratios on mixing and concluded that the velocity ratio is the most important to determine the near and far field mixing of coaxial jets. Champagne and Wygnanski [5] used a hot-wire anemometer in their investigation of coaxial turbulent jets. Champagne and Wygnanski pointed out that the quantities defining the configuration, and thus potentially influencing the characteristics of varies flow regions, of a coaxial jet are numerous; the inner and outer exit velocity, $U_{i}$ and $U_{o}$, the inner and annular areas, $A_{i}$ and $A_{0}$. Two jets are used with area ratios of $\mathrm{A}_{\mathrm{o}} / \mathrm{A}_{\mathrm{i}}=0.94$ and 1.28 with the corresponding Reynolds numbers ranging from 0.0 to $10^{5}$ at velocity ratio variation of $0.0 \leq \mathrm{U}_{\mathrm{o}} / \mathrm{U}_{\mathrm{i}} \leq 10.0$. The internal jet is never completely stopped to prevent the creation of an angular jet with its low-pressure recirculating bubble through which the hot wire cannot measure accurately as it cannot distinguish the reversed flow.

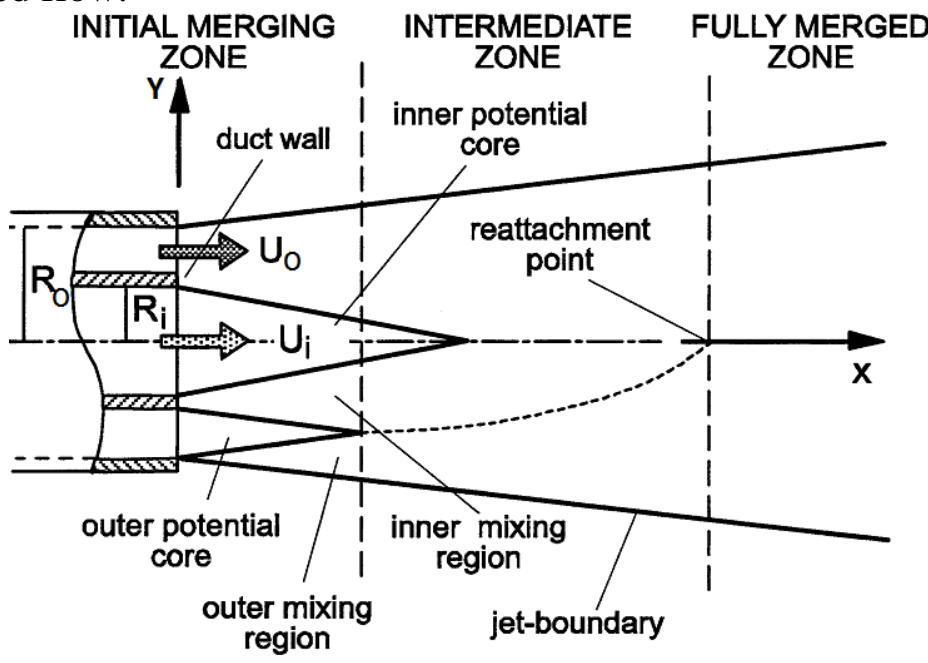

(a) 


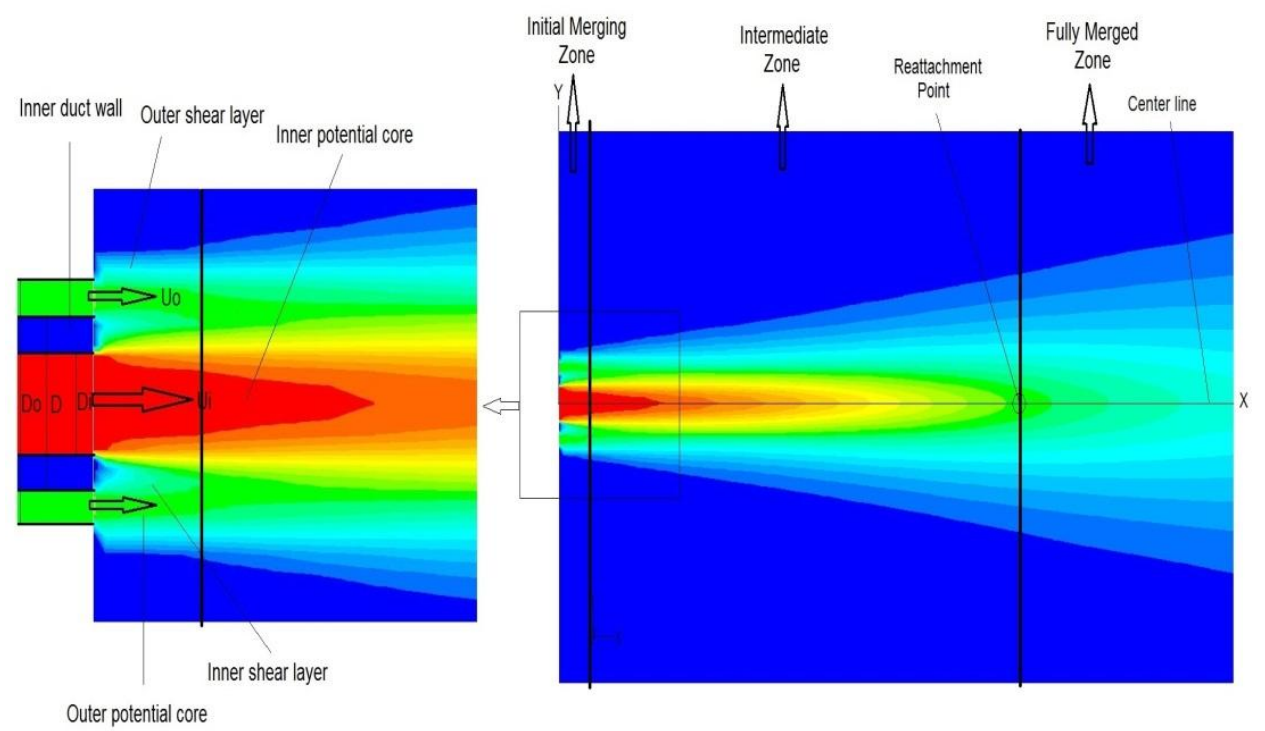

(b)

Fig. 1: (a) Flow field of a coaxial jet configuration, schematically [7,9] and (b) coaxial jet configuration by CFD simulations

Combustion reactions are commonly diffusion-controlled, i.e. the kinetics of the reaction is so rapid that the rate of reaction is completely determined by the turbulent mixing of the reactants. Stark [6] experimentally investigated the flow field in a flame formed by coaxial jet for a range of outer to inner diameter from 1.9 to 3.8 while varying the initial velocity of the outer jet to that of the inner jet by a factor of $3: 1$. Total head measurements have been made and the mean velocity profiles are presented for various axial distances up to eighteen jet diameters downstream. The results are complicated by the presence of temperature and density variation caused by the flame and the rather thick boundary layers in the nozzles. Stark reported that the initial velocity condition of the inner jet is a fully developed turbulent pipe flow. The initial annular separation of the jets leads to a separation bubble which makes the flow field quite complex and further makes the results valid only for their particular nozzle design.

More recent studies using Laser Doppler Anemometry (LDA) such as Buresti et al. [7], Buresti et al. [8] and Warda et al. [9] proposed a significant data base for a particular coaxial jet configuration. This base provides mean axial velocities profiles, turbulence intensities and shearing tensions in initial and intermediate regions. These authors took typical turbulence characteristics of industrial applications such as premixed burners. They specified the quantities that used to define the configuration, and potentially affect the characteristics of the three regions of a coaxial jet. These quantities are numerous such as the inner and outer velocity ejection, the inner and annular surfaces, the inner nozzle wall thickness, the importance of the boundary layer thicknesses at the exit, as well as their state (laminar or turbulent) and the turbulence levels at the nozzle exit.

The mixing mechanisms associated with such coaxial jets are further elaborated by a later study of Villermaux and Rehab [10]. Along the numerical simulation front, Balarac and Me'tais [11] carried out a numerical study to investigate the role of nozzle lip thickness and found that smaller thicknesses tend to lead to faster transition to turbulence and affect the shape and extent of the recirculation bubble formed when the velocity-ratio is beyond the critical value. The effects of forcing upon coaxial jets are also studied by Balarac et al. [12], where the presence of excitations generally improves coaxial jet mixing characteristics and supported earlier findings obtained by Wicker and Eaton [13]. 
Manivannan et al. [14] reported that single non-circular jets have better mixing characteristics than their axi-symmetric counterparts. Accordingly, a much effort has been expended in understanding the fundamental mechanisms of jet mixing and applying such knowledge in nozzle modifications to achieve satisfactory mixing levels. Early forms of nozzle modification involved changing the nozzle geometry from conventional circular shape to noncircular shapes i.e., elliptic, rectangular, square, and others. For instance, Ho and Gutmark [15], Hussain and Husain [16], Zaman [17] and Grinstein and DeVore [18] investigated different nozzle geometries that achieve significantly better mixing and control over the circular geometry, mainly due to their inherent axis-switching behavior and formation of highly threedimensional vortical structures (such as braid and rib vortices) at the corners. More complicated lobed nozzles have been also considered by Hu et al. [19] and Zaman et al. [20] to produce strong coherent vortices aligned in the stream-wise direction to improve jet mixing abilities. These studies essentially show that jet vortex dynamics can be manipulated favorably in terms of mixing by altering the nozzle contour appropriately.

The effects of inlet elliptic and coaxial jet geometry on the mixing process with large density differences have been investigated numerically using a second-order Reynolds stress model (RSM) by Senouci et al. [21]. It was found that the predicted results agree reasonably well with the recently experimental data. In addition, the study shows that the performances of the elliptic geometries are much higher than those of the circular. As a result, the asymmetrical coaxial nozzles enhance strongly the mixing.

In modeling of circular and non-circular co-axial fuel injectors based on hydraulic diameter of single jet, Reddy et al. [22] show a good turbulence kinetic energy in non-circular shape compared to circular shape except circle-square one (proposed shapes). While, the turbulence characteristics such as turbulence eddy dissipation, turbulence kinetic energy, and velocity profiles along the axial length are not providing good turbulence kinetic energy and turbulence eddy dissipation in circle-square shape compared with circular coaxial jet. Whereas, the circle-hexagonal shape produces turbulence kinetic energy and turbulence eddy dissipation more than circular coaxial jet.

One of the many approaches toward further improvisations to any nozzle design in a rational manner lies in good appreciation of the underlying vortex structures and their associated behavior. To that end, a study by New et al. [23] was carried out to isolate the basic flow dynamics from relatively simple V-notched nozzles of moderate "sharpness". A vortex flow model based on flow visualizations has been proposed to explain the observed flow structures and how they are formed. Subsequently, hot-wire measurements by New and Tsai [24] have been also documented to understand the nature of the mean velocity and turbulence flow field produced by nozzle peaks and trough of different sharpness. The convoluted nature of the vortex structures shown in the limited flow visualization images presented in New et al. [23] raised the question of how different the vortex dynamics would be for relatively sharper nozzles. For instance, in the vortex flow model proposed in New et al. [23], which made use of results gathered on a moderately sharp V-notched nozzle, the stream-wise vortices are postulated to result from the distortions to the vortex lines due to the undulated nozzle-exit lip contour. In the case of a much sharper nozzle, accentuated vortex structures, increased selfinductions and augmented interactions between the various flow structures are expected to lead to observable flow discrepancies. To shed some light on these issues New [25], New and Tsovolos [26], Tsioli and New [27] and New and Tsovolos [28] studied this type of nozzle design.

In above contest, an extensive research has been carried on non-circular coaxial jet. However, these kinds of jets need more investigations to explore the shapes that give high performance. As a result, in the current study, different jet configurations have been studied. In this study, the flow evolves into a single jet after merging into the annular and inner flows. Also, the non-circular shape such as triangle for the inner nozzle and then for the outer one is used to study the flow behavior and jet flow characteristics. The length of potential core, jet decay 
and jet flow field characteristics at near field of the coaxial jet flow are the key parameters used to evaluate the coaxial jet performance.

\section{MTHEMATICAL MODEL}

\subsection{Governing Flow Equations}

The flow field is considered within the domain identified by Cartesian coordinates $\mathrm{x}$ (streamwise), and y, z (cross-stream) as indicated in Fig. 1(a \& b). The flow field is considered incompressible, steady, asymmetric and turbulent. The coaxial flow jet is modeled by solving the continuity and momentum equations which can be given as Strohel et al. [29]

\section{Continuity equation:}

$$
\nabla \cdot V=0
$$

\section{Momentum equation:}

$$
V . \nabla V=-\left(\frac{1}{\rho}\right)\left[\nabla P+\nabla \cdot\left[\left(\mu+\mu_{t}\right) \nabla V\right]\right]
$$

where $\rho$ is the density, $V$ is the Reynolds-averaged velocity vector, $P$ is the Reynoldsaveraged pressure, $\mu$ is the dynamic molecular viscosity, and $\mu_{t}$ is the eddy viscosity. The gravitational force is neglected in the current study.

Practically, turbulence plays an important role in the enhancement fuel mixing and leads to increase burning velocity. Therefore, in the present study the effect of nozzle geometry on the flow field is modeled using the two equations of Reynolds Average Navier-Stokes (RANS) turbulence models, standard $k-\varepsilon$ model [30]. This model has been used in many similar studies for example Stroher et al. [29], Berg et al. [31], Kriaa et al. [32] and Villermaux and Rehab [33] and gives satisfactory agreement with experimental data.

In this model, the eddy viscosity, $\mu_{t}$, is computed by

$$
\mu_{t}=\rho C_{\mu} \frac{k^{2}}{\varepsilon},
$$

where $k$ and $\varepsilon$ are the turbulent kinetic energy and dissipation energy, respectively and can be given by

$$
\begin{gathered}
\frac{\partial(\rho k)}{\partial t}+\frac{\partial\left(\rho k u_{i}\right)}{\partial x_{i}}=\frac{\partial}{\partial x_{j}}\left[\frac{\mu_{t}}{\sigma_{k}} \frac{\partial k}{\partial x_{j}}\right]+2 \mu_{t} E_{i j} E_{i j}-\rho \epsilon \\
\frac{\partial(\rho \epsilon)}{\partial t}+\frac{\partial\left(\rho \epsilon u_{i}\right)}{\partial x_{i}}=\frac{\partial}{\partial x_{j}}\left[\frac{\mu_{t}}{\sigma_{\epsilon}} \frac{\partial \epsilon}{\partial x_{j}}\right]+C_{1 \epsilon} \frac{\epsilon}{k} 2 \mu_{t} E_{i j} E_{i j}-C_{2 \epsilon} \rho \frac{\epsilon^{2}}{k}
\end{gathered}
$$

, respectively, where $u_{i}$ the velocity component in corresponding direction is, $E_{i j}$ is component of rate of deformation. In addition to some adjustable constants with default values of $\sigma_{k}=1.0, \sigma_{\varepsilon}=1.3, C_{1 \varepsilon}=1.44$ and $C_{2 \varepsilon}=1.92$ are used.

\subsection{Computational Model}

Four different flow patterns have been used in the current study. The first is a coaxial jet of Durao and Whitelaw [34], shown in Fig. 2, which has been used for the numerical validation purpose. 


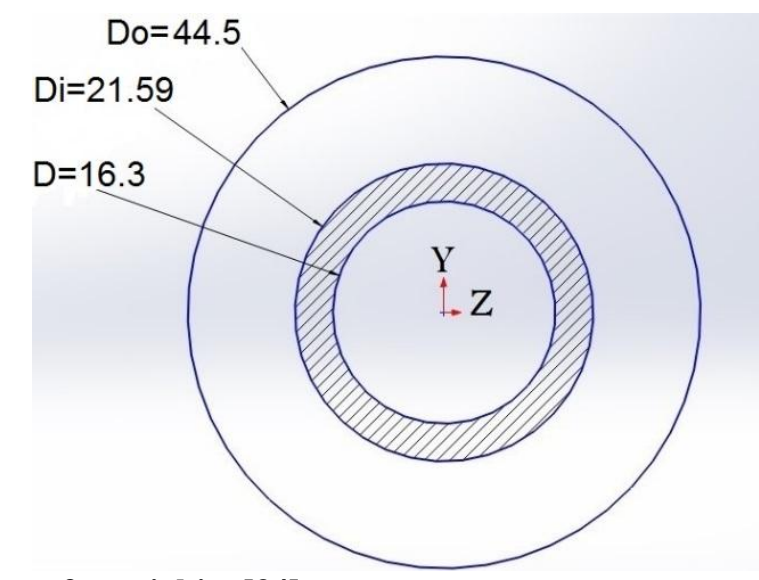

Fig. 2: circular configuration of coaxial jet [34].

The dimensions of the computational domain, which are defined by $Y=Z=31 \times D$ while $0 \leq X \leq 62 \times D$, and boundary conditions are shown in Fig. 3(a \& b). No slip condition is adopted during the computational process. The boundary condition at face (1) is a velocity inlet (uniform velocity), face (2) is a wall and face (3) is atmospheric pressure. In this case, the grid is used as $8800 \times 50$ cells with stretching in both radial and stream-wise direction, respectively.

a)

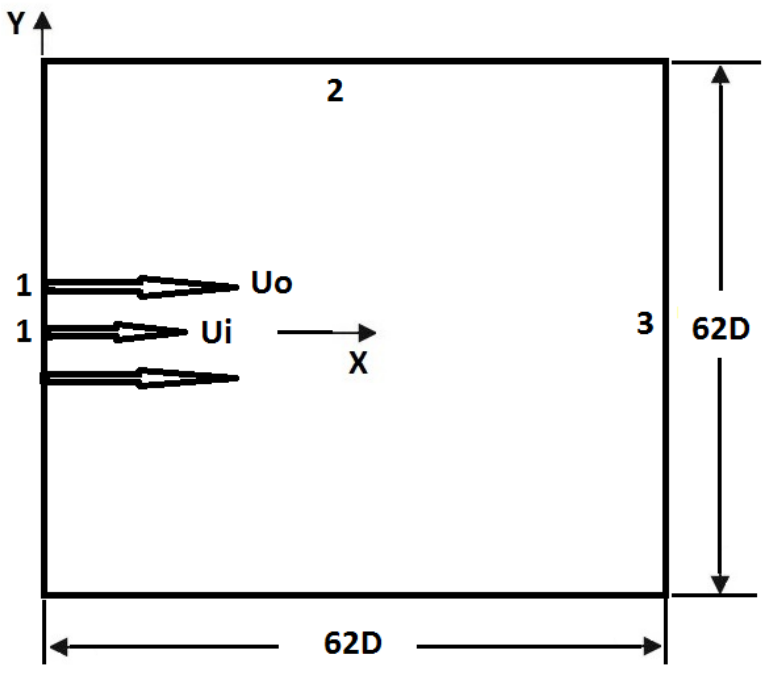

b)

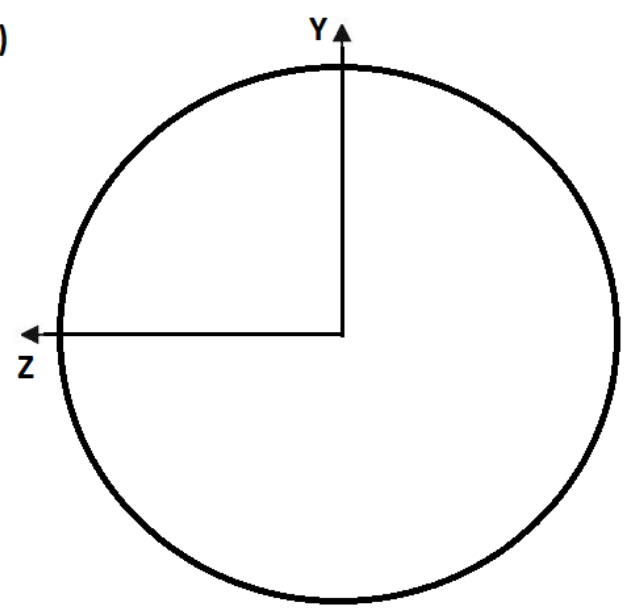

Fig. 3(a) Elevation section included the faces number; (b) Side view.

In addition, the current work is extended to include the other three jet configurations, shown in Fig. 4. These configurations are formed by two different nozzle shapes namely circular and triangular cross sections, in three combinations: Case-0 [circle (outer)-cirlce (inner)], Case-1 [circle (outer)-triangle (inner)] and Case-2 [triangle (outer)-circle (inner)]. 

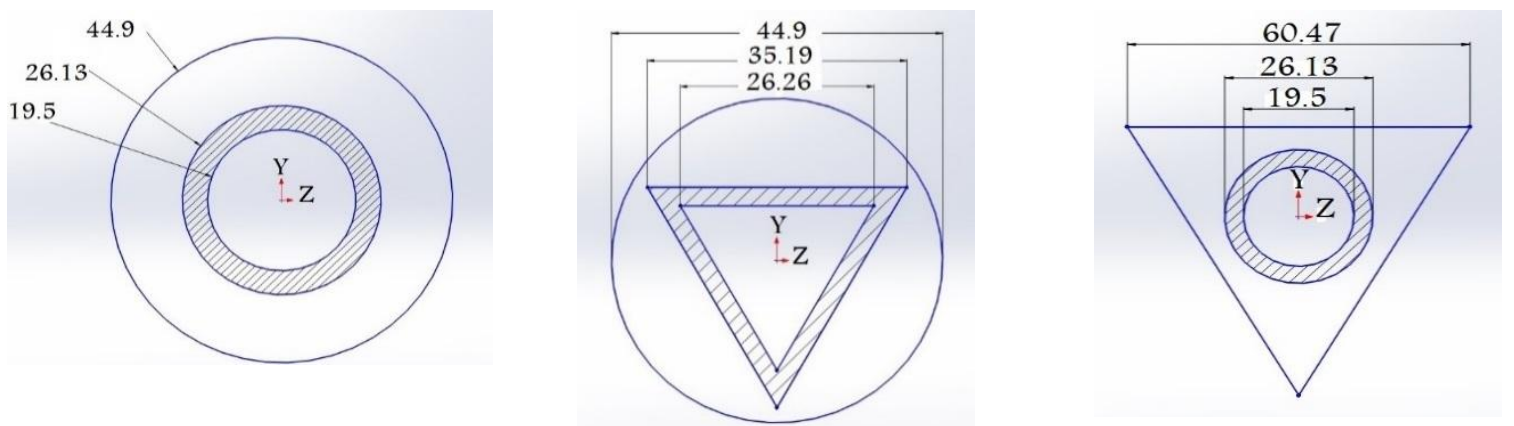

Fig. 4: Geometries of coaxial jet studied in the current work

The dimensions in each jet are set in such a way to yield the same equivalent diameter, $D_{e q}=B \sqrt{\frac{\sqrt{3}}{\pi}}=L \frac{2}{\sqrt{\pi}} \quad(B$ and $L$ are triangle and square ribs, respectively $)$. The shape and thickness of the separating wall are not the same in these cases, but depend on the geometry of the two coaxial jet, for example: Case-1 (Fig. 2-b) the thickness of the separating wall is $2.6 \mathrm{~mm}$, whereas in Case-0 and Case- 2 is $3.3 \mathrm{~mm}$; to maintain the area ratio, $A R$, which equals $=\frac{A_{o}-A_{i}}{A}, A_{o}=\frac{\pi}{4} D_{o}{ }^{2}, A_{i}=\frac{\pi}{4} D_{i}{ }^{2}$ and $A=\frac{\pi}{4} D^{2}$, as $\mathrm{AR}=3.5$.

The corners in the non-circular shapes are rounded by radius $2 \mathrm{~mm}$ which isn't taken into consideration in the previous investigation. The air inside the domain into which the jet flow is emerging, is quiescent. The coordinate system and computational domain are indicated in Fig. 5. The inlet conditions specified in all cases are listed in table 1.

\subsection{Numerical Solution}

The flow field was calculated by solving the Reynolds Averaged Navier Stokes equations (RANS) and turbulence models equations available in a CFD package (FLUENT 6.3). The equations are discretized by finite volume method and solved using the "uncoupled" solver and convective terms discretized using the spatial second order scheme, Barth and Jespersen [35]. The pressure-velocity coupling algorithm used is SIMPLE. The wall has been treated in turbulent flow as standard wall function. Solutions were considered converged when the maximum residual of all the discretized equations is lower than $1 \times 10^{-5}$. Structured and non-uniform grids are generated for the solution domain, shown in Fig. 6.

a)

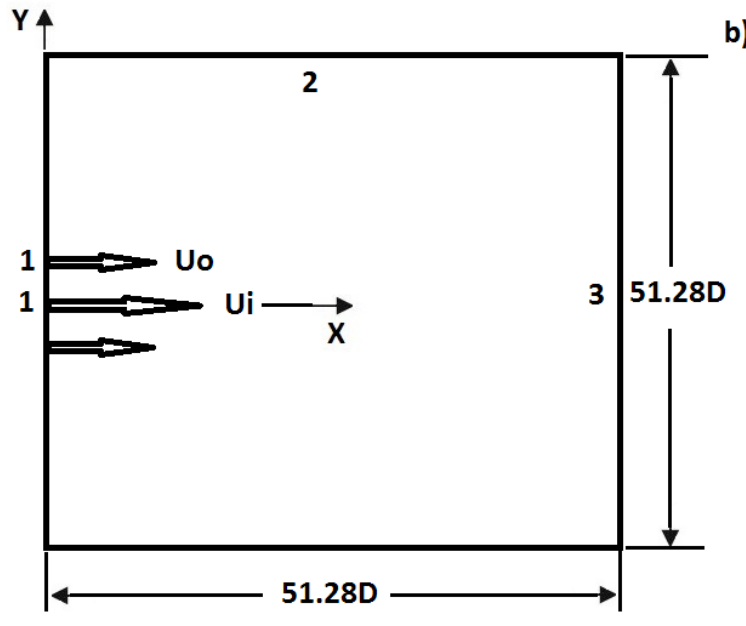

b)

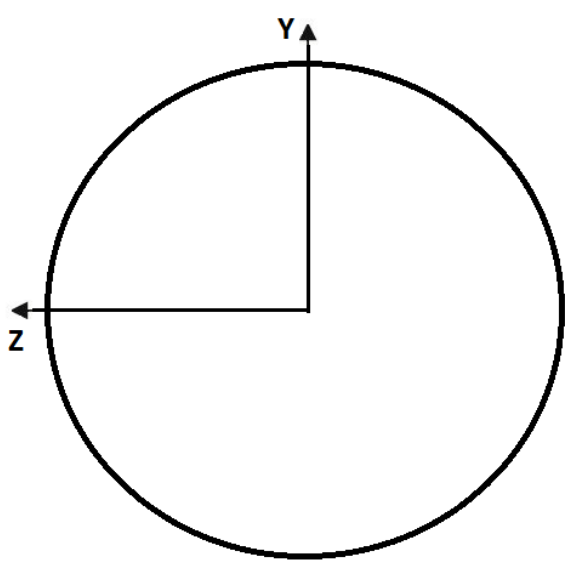

Fig. 5: (a) Elevation section; (b) Side view.

Table 1: Inlet conditions of validation and present cases. 


\begin{tabular}{|c|c|c|c|c|}
\hline & Inner stream & Outer stream & Inner stream & Outer stream \\
\hline (2) & $\begin{array}{l}y-2 \\
x-2 \\
\end{array}$ & & (c) & $\frac{M N}{M+1}$ \\
\hline Velocity $[\mathrm{U}] \mathrm{m} / \mathrm{s}$ & 25.077 & 40.447 & 60 & 30 \\
\hline Equivalent diameter $\left[D_{\text {eq }}\right] \mathrm{mm}$ & 16.13 & 38.9 & 19.5 & 36.51 \\
\hline Reynolds number $[R e]=\frac{\rho U D_{e q}}{\mu}$ & $2.77 \times 10^{4}$ & $10.8 \times 10^{4}$ & $8 \times 10^{4}$ & $7.5 \times 10^{4}$ \\
\hline $\begin{array}{l}\text { Turbulence } \\
{[I]=0.16 \operatorname{Re}^{-1 / 8} \%}\end{array}$ & 4.45 & 4.02 & 3.90 & 3.93 \\
\hline Mach number & 0.072 & 0.117 & 0.173 & 0.087 \\
\hline
\end{tabular}

Fig. 6: Detailed view of 3D grid for exit nozzle.

Mesh independence test are performed by using three computational grid levels as: Mesh$1=4284 \times 50$ (coarse), Mesh-2=5312x50 (medium), and Mesh-3=6414x5030 (fine). The grid independent study is performed on the circular coaxial jet (Case-0) with the flow conditions, indicated in Table 1. This study is shown in Fig. 7 in terms of pressure ratio (PC/PO) where $\mathrm{Pc}$ is total pressure in the axial direction and Po is the pressure at the nozzle exit plane. It is found that the maximum percentage change in result is $1.5 \%$. Therefore, Mesh-3 $(2.5 \mathrm{~mm}$ edge interval size) has been chosen for all subsequent computations.

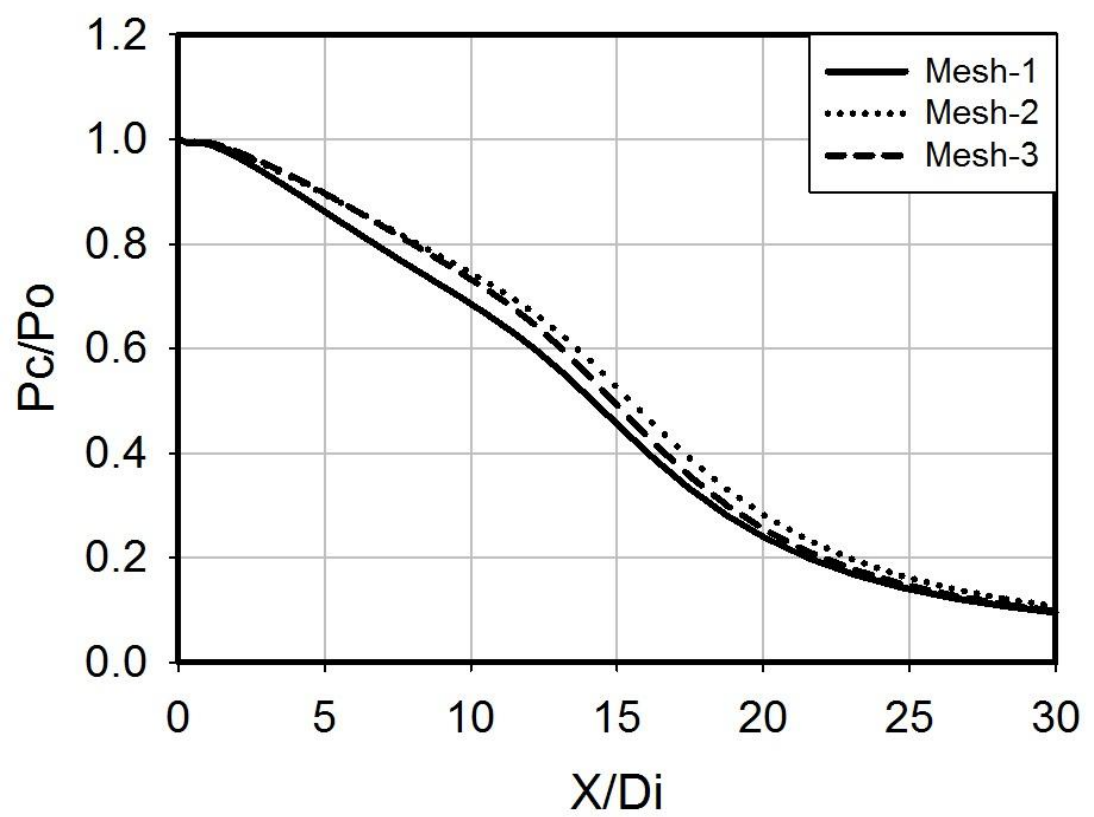

Fig. 7: Grid independent study. 


\section{RESULTS AND DESCUSSION}

Figures $8(\mathrm{a} \& \mathrm{~b})$ show the predicted mean velocity along the co-axial jet centerline and in radial direction as a function of $\mathrm{X} / \mathrm{D}_{\mathrm{i}}$ and $\mathrm{Y} / \mathrm{D}_{\mathrm{i}}$, respectively compared with the experimental data obtained by Durao and Whitelaw [34]. The results are, to some degree, in a reasonably good agreement with experiment. As a result, the computational method used in this study is verified.

(a)

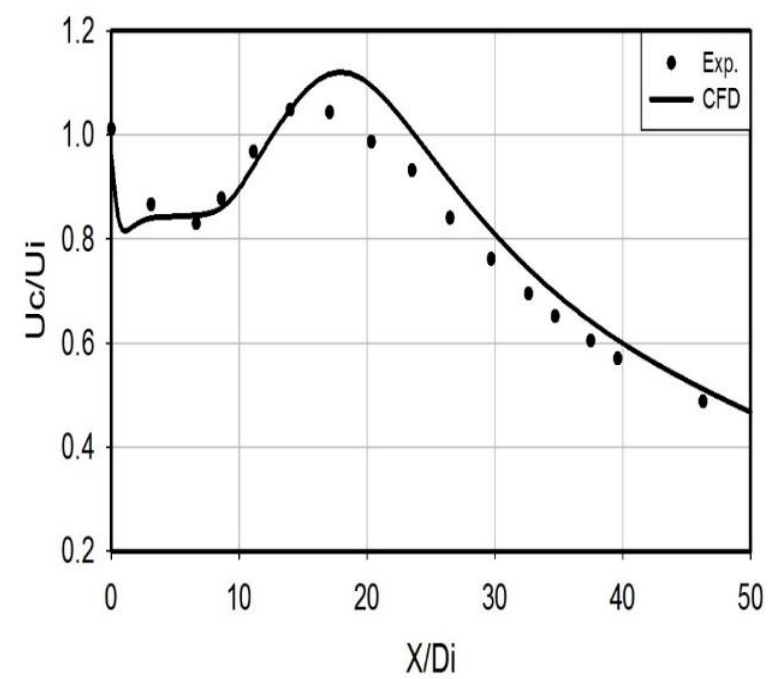

(b)

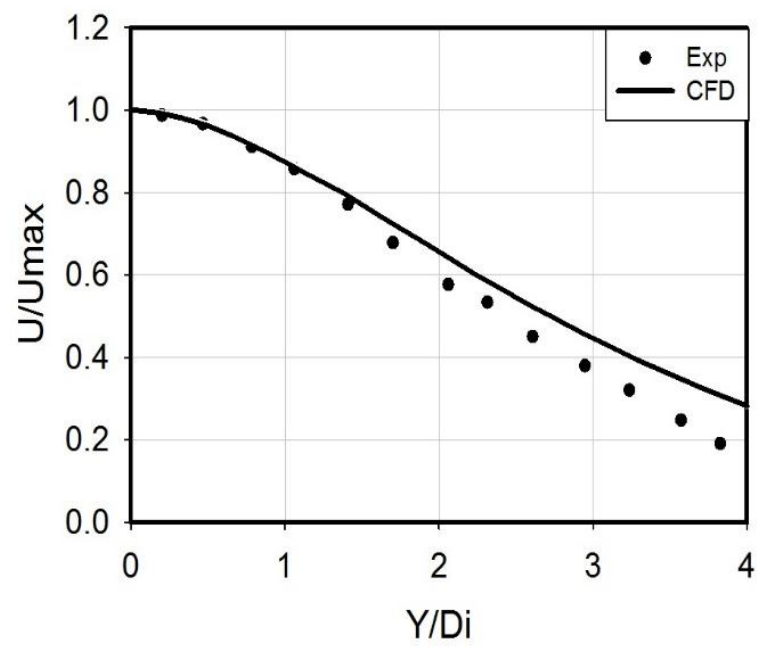

Fig. 8: Comparison of experimental and predicted results for (a) the decay along the coaxial jet centerline and (b) the decay in radial direction at $\mathrm{X}=10.86 \mathrm{Di}$

The cases, shown in Fig. 4, have been performed for an incompressible coaxial jet. The coaxial jet orifices of different geometries like circle and equilateral triangle form have been used during these cases with constant velocity ratio, $\mathrm{U}_{\mathrm{o}} / \mathrm{U}_{\mathrm{i}}$ of 0.5 . A computational data procured at different axial locations are presented in Figs. 9-12 and discussed below.

\subsection{Jet Decay studies-using centreline velocity profiles}

The centerline velocity decay, defined as ratio between the centerline velocity and core jet inlet velocity $\left(\mathrm{U}_{\mathrm{c}} / \mathrm{U}_{\mathrm{i}}\right)$, is shown in Fig. 9. The velocity decay of Case-0 (circular) is comparatively higher than Case-1 and Case-2 (non-circular), but it has the lowest rate of decay.

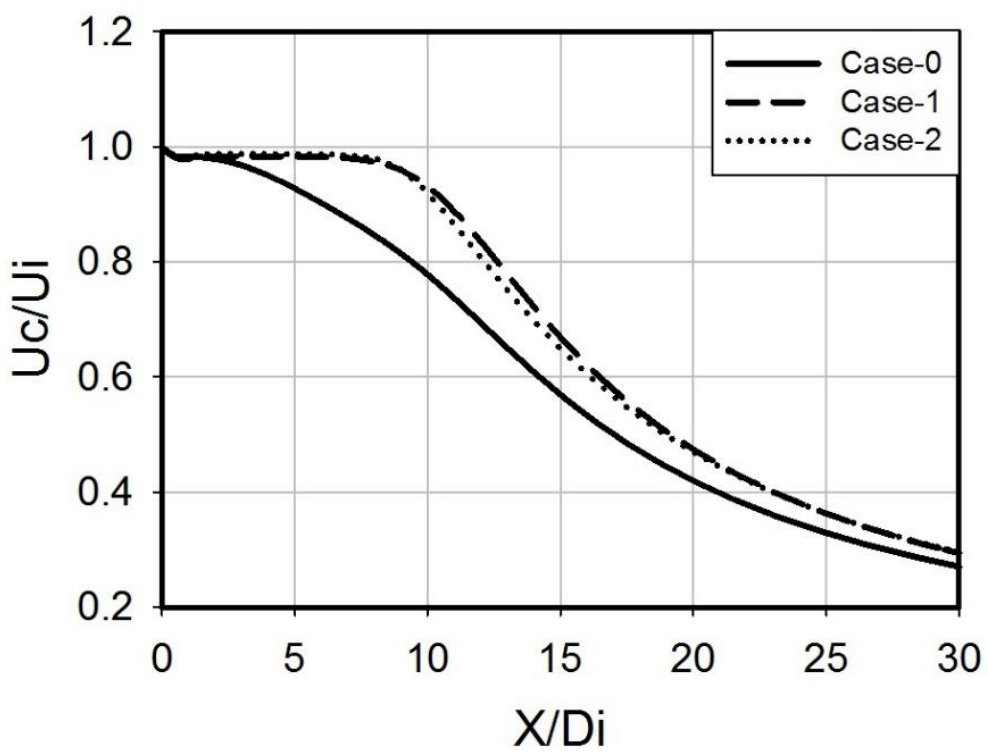

Fig. 9: The decay velocity along the centerline for different configurations of coaxial jet 
At the nozzle exit the flow consists of two potential cores (The potential core, inner or outer, which is defined as the region in which the mean axial velocity equal to the mean exit velocity from the nozzle, and its length which is the length of this region in X-direction) and two mixing regions as indicated in Fig. 1(b). The width of each core decreases with downstream distance. The length of the external core appears to be independent of the initial velocity ratio $\mathrm{U}_{\mathrm{o}} / \mathrm{U}_{\mathrm{i}}$. While, the length of the inner core strongly depends on $\mathrm{U}_{\mathrm{o}} / \mathrm{U}_{\mathrm{i}}$, as well as on the area ratio [5]. The obtained lengths of outer and inner potential core are listed in Table 2 . The jet velocity is relatively constant inside the potential core of the jet as revealed in Fig. 1(a and b). Therefore, according to Fig. 9 and data listed in Table 2, the potential core length of the three co-axial jets, Case- 0 , Case- 1 , and Case- 2 , are about $3 \mathrm{D}_{\mathrm{i}}$, $8 \mathrm{D}_{\mathrm{i}}$ and $8 \mathrm{D}_{\mathrm{i}}$, respectively. The non-circular coaxial jet has longer inner potential core than the circular coaxial jet.

Table 2. The lengths of wake zone and inner- and outer-potential cores and location of reattachment point.

\begin{tabular}{lccc}
\hline \multicolumn{1}{c}{ Property } & Case-0 & Case-1 & Case-2 \\
\hline Length of outer potential core (X/D) & 1.3 & 3.7 & 0.5 \\
Length of inner potential core (X/D) & 3 & 8 & 8 \\
Reattachment point location (X/D) & 16 & 19 & 18 \\
Length of wake region (X/D) & 1 & 3 & 0.4 \\
\hline
\end{tabular}

\subsection{The reattachment point}

In the fully merged region, as named by Ko and Kwan [1], the centerline velocity is much reduced, the radial velocity profiles developed into bell-shaped distributions and the fully merged flow behaved like a combined (single) jet [9]. This region begins just after the reattachment point which, shown Fig. 1 (a) [7 \& 9], can be obtained from the intersection of parabolic contour of outer jet velocity, dotted line in Fig. 1(a) and green color in Fig. 10, and the coaxial jet centerline.

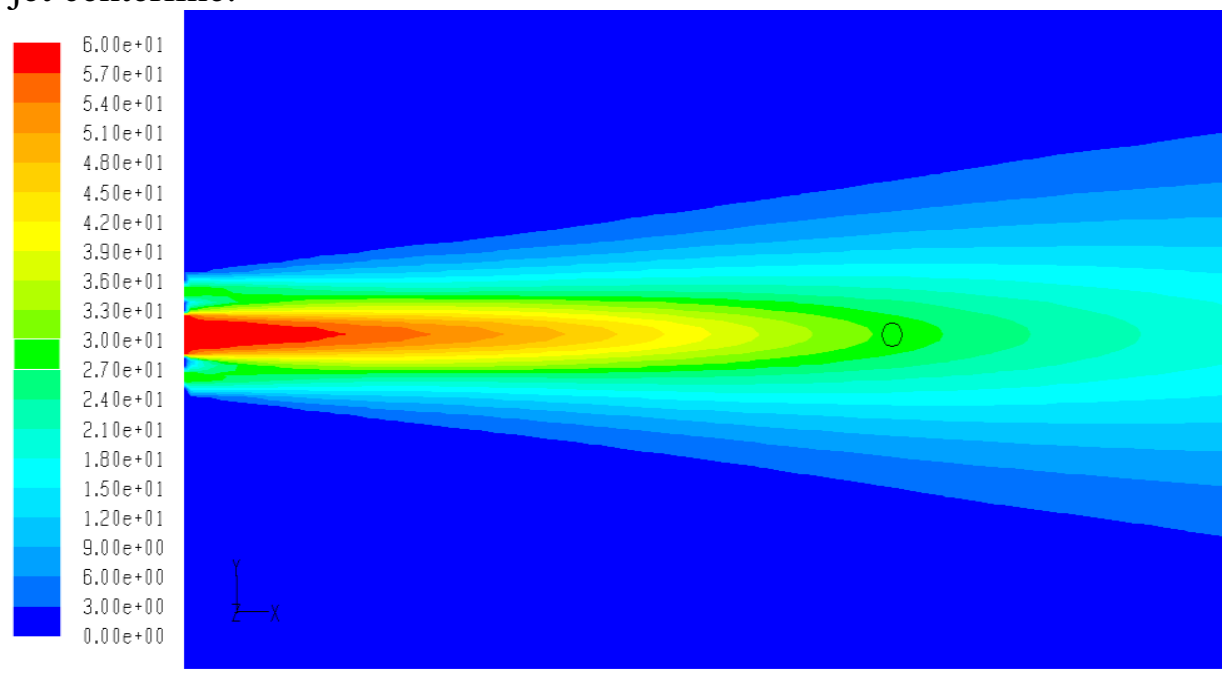

Fig. 10: the velocity contours of case-0.

In Table 2, the locations of reattachment point for Case- 0 , Case- 1 and Case- 2 are listed at approximately $\mathrm{X} / \mathrm{D}_{\mathrm{i}}=16,19$ and 18 , respectively. These results mean that the mixing between the two streams in circular coaxial jet occurs faster than non-circular jets.

\subsection{Effect of jet shapes on velocity distributions along the radial (Y) direction:}

The velocity distributions in the radial $(\mathrm{Y})$ direction for different configurations are illustrated in Fig. 11. The velocity decay of circular coaxial jet (Case-0) along the radial direction is less 
than the non-circular coaxial jet (Case-1 and Case-2). The velocity decay in triangular outer nozzle co-flow jet is faster than the circular outer nozzle coaxial jet. This is due to the corner vortices in outer shear layer. In addition, from the radial profiles of velocity distribution, the length of the wake region which resulted from the inner nozzle thickness, shown in Fig. 12, can be calculated (where the wake region is defined as the region in which the back flow appear, and its length is from the nozzle exit plane to the end of this region in X-direction). The lengths of wake zone, inner- and outer-potential cores as well as the location of reattachment point as a function of X/Di are listed in Table 2.

(a)

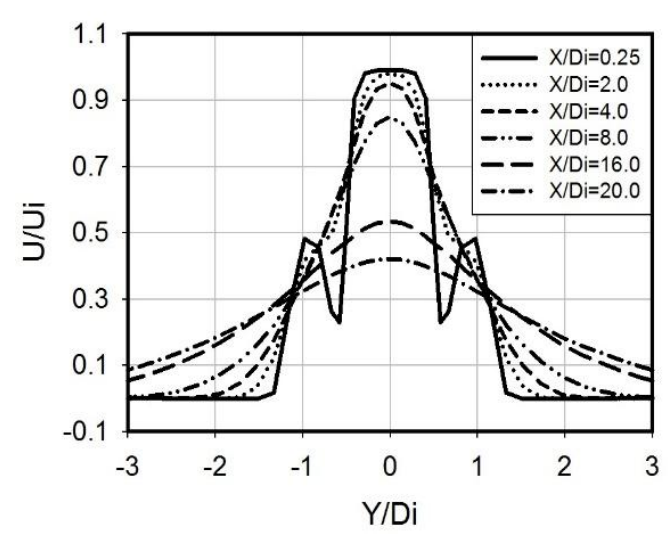

(b)

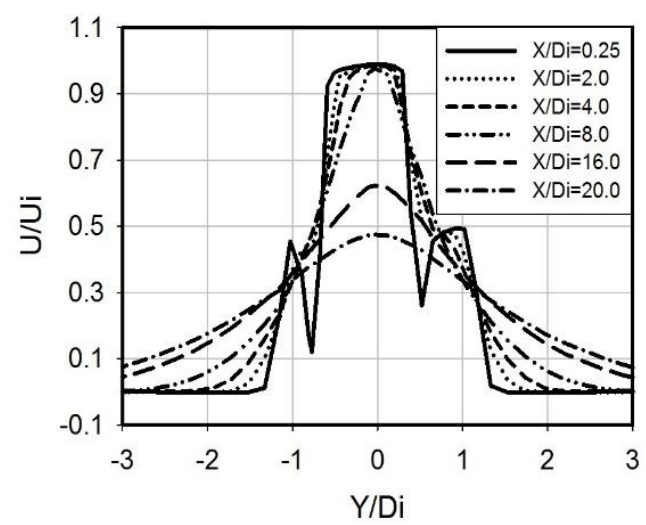

(c)

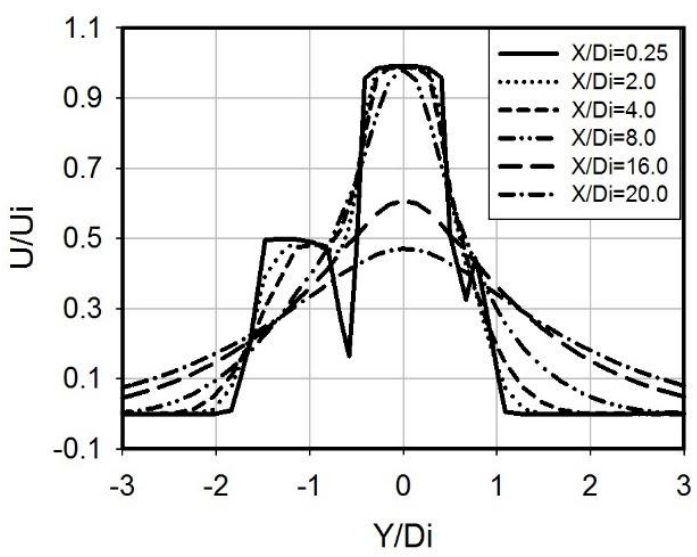

Fig. 11: Radial profiles of the axial mean velocity of a coaxial jet, (a) case- 0 , (b) case- 1 and (c) case-2.

\section{4. conclusion}

Based on numerical investigations of an incompressible circular and non-circular coaxial jet using triangle geometry for outer and inner nozzle, the following conclusions have been drawn:

- The velocity decay along the centerline of circular co-axial jet is comparatively higher than non-circular ones, but with lower rate.

- The potential core length of circular coaxial jet is less than the non-circular co-flow jets and same for both non-circular cases.

- The non-circular geometries have a significant effect on specifying the reattachment point, where circular coaxial jet is the first one in reaching the self-similarity at $16 \mathrm{D}$ and the triangular jet with circular outer pipe is the last which attained at $19 \mathrm{Di}$. 
- The velocity decay rate along the radial direction of non-circular coaxial jet is also higher than the circular coaxial jets.

- The size of wake region is minimum in case- 2 and maximum in case- 1

\section{References}

[1] Ko, N.M.W. and Kwan, A.S.H., "The initial region of subsonic coaxial jets,” J. Fluid Mech. 73 (1976) 305-332.

[2] Ablitzer C., Gray F., and, Perrais C., "Influence of macromixing on powder formation in a reactive coaxial jet," Chemical Engineering Science 57 (2002) 2837-2848.

[3] Pope, S. B., “Turbulent Flows,” Cambridge University Press, Cambridge, GB (2000).

[4] Forstall, W. and Shapiro, A.H., "Momentum and mass transfer in coaxial gas jets," Trans. ASME. J. Appl. Mech. 18 (1951) 219-228.

[5] Champagne, F.H. and Wygnanski, I.J., "An experimental investigation of coaxial turbulent jets,” Int. J. Heat and Mass Transfer 14 (1971) 1445-1464.

[6] Stark, S.B., "Mixing of gas streams in a flame,” Zh. Tekh. 23 (1953) 1802-1819.

[7] Buresti, G., Talamelli, A. and Petagna, B., "Experimental characterization of the velocity field of a coaxial jet configuration,” Exp. Therm. Fluid Sci. 9 (1994) 135-146.

[8] Buresti, G., Petagna, B. and Talamelli, A., "Experimental investigation on the turbulent near field of coaxial jets," Experimental Thermal and Fluid Science 17 (1998) 18-36.

[9] Warda, H.A., Kassab, S.Z., Elshorbagy, K.A. and Elsaadawy, E.A., “An experimental investigation of the near-field region of a free turbulent coaxial jet using LDA," Flow Measurement and Instrumentation. 10 (1999) 15-26.

[10] Villermaux, E. and Rehab, H., "Mixing in coaxial jets," J. Fluid Mech. 425 (2000) 161185.

[11] Balarac, G. and Métais, O., "The near field of coaxial jets: a numerical study," Phys. Fluids 17 (2005) 065-102.

[12] Balarac, G., Métais, O. and Lesieur, M., "Mixing enhancement in coaxial jets through inflow forcing: a numerical study," Phys. Fluids 19 (2007) 075-102.

[13] Wicker, R.B. and Eaton, J.K., "Near field of a coaxial jet with and without axial excitation," AIAA J. 32 (1994) 542-546. 
[14] Manivannan, P. Dash, P.K. and Sridhar, P.T.N., “An Experimental Study on Comparison of Non-circular Co-flow Jet with Co-axial Jets and Computational Verification,” Space Research Journal. 4(2) (2011) 60-70.

[15] Ho, C.M., and Gutmark, E., "Vortex induction and mass entrainment in a small-aspectratio elliptic jet,” J. Fluid Mech.179 (1987) 383-405.

[16] Hussain, F., and Husain, H.S., "Elliptic jets. Part 1. Characteristics of unexcited and excited jets," J. Fluid Mech.208 (1989) 257-320.

[17] Zaman, K.B.M.Q., “Axis switching and spreading of an asymmetric jet: The role of coherent structure dynamics,” J. Fluid Mech. 316 (1996) 1-17.

[18] Grinstein, F.F. and DeVore, C.R., "Dynamics of coherent structures and transition to turbulence in free square jets," Phys. Fluids 8 (1996) 1237-1251.

[19] Hu, H., Saga, T., Kobayashi, T. and Taniguchi, N., “A study on a lobed jet mixing flow by using stereoscopic particle image velocimetry technique," Phys. Fluids 13 (2001) 3425-3441.

[20] Zaman, K., Wang, F.Y. and Georgiadis, N.J., "Noise, turbulence and thrust of subsonic free jets from lobed nozzles,” AIAA J. 41 (2003) 398-407.

[21] Senouci, M., Belkadi, M., Bouguenina, B. and Imine, B., "Numerical study of elliptic and coaxial jets with variable density," MECHANIKA. 18(5) (2012) 534-538.

[22] Narasimha Reddy, N.L., Manivannan, P. and Kiran Babu, K.M., "The CFD Analysis of Turbulence Characteristics in Combustion Chamber with Non Circular Co-Axial Jets," Journal of Mechanical and Civil Engineering 6(2) 2013 1-10.

[23] New, T.H., Lim, K.M.K. and Tsai, H.M., "Vortical structures in a laminar V-notched indeterminate-origin jet," Phys. Fluids 17 (2005) 054-108.

[24] New, T.H. and Tsai, H.M., "Experimental investigations on in determinate origin Vand A-notched jets," AIAA J.45 (2007) 828-839.

[25] New, T.H., "An experimental study on jets issuing from elliptic inclined nozzles," Exp. Fluids 46 (2009) 1139-1157.

[26] New, T.H. and Tsovolos, D., "Influence of nozzle sharpness on the flow fields of Vnotched nozzle jets," Phys. Fluids 21 (2009) 084-107. 
[27] Tsioli, E. and New, T.H., "Near-field vortex structures of inclined coaxial jets," Proceedings of sixth international symposium on turbulence and shear flow phenomena, Seoul, Korea (2009) 499-504.

[28] New, T.H. and Tsovolos, D., "On the vortical structures and behavior of inclined elliptic jets. Eur. J. Mech. B, Fluid 30 (2011) 437-450.

[29] Stroher, G.R., Martins, C.A. and de Andrade, C.R., "Numerical and Experimental Study of a free incompressible isothermal turbulent coaxial Jet," Thermal Engineering, 9 (2010) 98-107.

[30] Jones, W. P. and Launder, B. E., "The Prediction of Linearization with a. Two-Equation Model of Turbulence,” International Journal of Heat and Mass Transfer. 15 (1972) 301314.

[31] Berg, J.R., Ormiston, S.J. and Soliman, H.m, "Prediction of the flow structure in a turbulent rectangular free jet," International Communications in Heat and Mass Transfer 33 (2006) 552-563.

[32] Wassim Kriaa, Kamel Abderrazak, Hatem Mhiri, Georges Le Palec and Philippe Bourno," A numerical study of non-isothermal turbulent coaxial jets," Heat Mass Transfer, 44 (2008) 1051-1063.

[33] Villermaux, E. and Rehab, H., "Mixing in coaxial jets," J. Fluid Mech. 425 (2000) 161185.

[34] Durao, D., and Whitelaw, J. H., "Turbulent Mixing in the Developing Region of Coaxial Jets,” Trans ASME, J. Fluids Engng. 95 (1973) 467-473.

[35] Barth T. J., and Jespersen D., "The design and application of upwind schemes on unstructured meshes,” 27th Aerospace Sciences Meeting, Reno, Nevada. (1989) Technical Report AIAA-89-0366. 